Article

\title{
Physiological and Transcriptome Analysis of a Yellow-Green Leaf Mutant in Birch (Betula platyphylla $\times$ B. Pendula)
}

\author{
Huixin Gang, Guifeng Liu, Su Chen $\$ and Jing Jiang * \\ State Key Laboratory of Tree Genetics and Breeding, Northeast Forestry University, 26 Hexing Road, \\ Harbin 150040, China; gang_em@163.com (H.G.); liuguifeng@126.com (G.L.); chensunefu@163.com (S.C.) \\ * Correspondence: jiangjing@nefu.edu.cn; Tel.: +86-139-4602-6246
}

Received: 19 December 2018; Accepted: 1 February 2019; Published: 2 February 2019

\begin{abstract}
Chlorophyll (Chl)-deficient mutants are ideal materials for the study of Chl biosynthesis, chloroplast development, and photosynthesis. Although the genes encoding key enzymes related to Chl biosynthesis have been well-characterized in herbaceous plants, rice (Oryza sativa L.), Arabidopsis (Arabidopsis thaliana), and maize (Zea mays L.), yellow-green leaf mutants have not yet been fully studied in tree species. In this work, we explored the molecular mechanism of the leaf color formation in a yellow-green leaf mutant $(y l)$. We investigated the differentially expressed genes (DEGs) between $y l$ and control plants (wild type birch (WT) and BpCCR1 overexpression line 11, (C11)) by transcriptome sequencing. Approximately 1163 genes ( 874 down-regulated and 289 up-regulated) and 930 genes (755 down-regulated and 175 up-regulated) were found to be differentially expressed in $y l$ compared with WT and C11, respectively. Kyoto Encyclopedia of Genes and Genomes (KEGG) pathway enrichment analysis for DEGs revealed that photosynthesis antenna proteins represent the most significant enriched pathway. The expressions of photosynthesis antenna proteins are crucial to the leaf color formation in $y l$. We also found that $\mathrm{Chl}$ accumulate, leaf anatomical structure, photosynthesis, and growth were affected in $y l$. Taken together, our results not only provide the difference of phenomenal, physiological, and gene expression characteristics in leaves between $y l$ mutant and control plants, but also provide a new insight into the mutation underlying the chlorotic leaf phenotype in birch.
\end{abstract}

Keywords: yellow-green leaf mutant; transcriptome; antenna protein; photosynthesis; birch

\section{Introduction}

Birch (Betula), a member of the tall deciduous tree family of Betulaceae, contains approximately 20 taxa birch species. Birch is an ecologically important tree species native to parts of China, Siberia, Korea, Japan, and Russia that has been introduced to many northern areas of the world [1]. This species grows fast and has a high tolerance that allows it to be used for revegetation and reforestation [2]. Birch also plays an important role in the forestry industry as a source of timber, fuelwood, plywood, pulpwood, and furniture [3-6]. Recently, there has been an increased interest in plants with colored leaves. Betula pendula 'Purple Rain', an intraspecific variety of B. pendula, has been used as a decorative plant for its purple leaves [7]. Previously, a yellow-green leaf mutant $(y l)$ was derived from cinnamoyl-CoA reductase $(B p C C R 1)$-overexpressing transgenic birch plants. The $y l$ mutant displayed a distinct yellow-green phenotype, while the leaves of all the other BpCCR1-overexpressing lines were a normal color, the same as the wild type birch (WT). One of the BpCCR1-overexpressing lines, C11 with green leaves, was used as control birch [8]. The $y l$ mutant is a very valuable resource because a yellow-green leaf is one of the most popular traits in landscape greening. 
Leaf color variation is one of the most common mutated traits as it is easily discovered in higher plants. Up to now, many Chl-deficient mutants have been identified in herbaceous and woody plants, including Arabidopsis [9], rice [10], maize [11], wheat (Triticum aestivum L.) [12], cotton (Gossypium barbadense L.) [13], and tea (Camellia sinensis (L.) O. Kuntze) [14]. It has been reported that a yellow-green leaf mutant (siygl1) of foxtail millet (Setaria italic L.) isolated following ethyl methanesulfonate (EMS) treatment was due to the loss function of the SiYGL1 gene. The identification of the SiYGL1 gene that encodes Mg-chelatase ATPase subunit D facilitated the understanding of the biological processes of chlorophyll (Chl) biosynthesis in millet [15]. Another yellow-green leaf mutant (ygl8) was proved to be controlled by the $Y g l 8$ gene, which encodes a chloroplast-targeted uridine monophosphate (UMP) kinase and affects chloroplast development in rice [16]. Therefore, Chl-deficient mutants are valuable genetic materials for exploring the molecular mechanisms of Chl biosynthesis and regulation, chloroplast development, plastid-to-nucleus signal transduction, and photosynthesis.

Next-generation sequencing (NGS) technologies have been considered as powerful tools for advanced research in many areas. Examples are genome and transcriptome sequencing of animals, plants, and microbes with high-throughput, high-speed, and high-accuracy sequencing data [17-19]. Wang, using transcriptome sequencing, analyzed a Chl-deficient chlorina tea plant culticar and reported the molecular mechanisms of the chlorine tea phenotype [14]. Study of a Lagerstroemia indica yellow leaf mutant using transcriptome analysis revealed the formation pathway of a yellow leaf mutant and discovered novel candidate genes related to leaf color [20]. The development of NGS technologies has increased the rate and efficiency of gene discovery and permitted a deeper understanding of the gene expression network.

In this work, we performed transcriptome sequencing for $y l$ with a yellow-green leaf phenotype, BpCCR1 overexpression line 11(C11), and wild type birch (WT) with a normal green leaf phenotype to analyze gene expression in these plants and elucidate the molecular mechanisms related to the different phenotypes. In addition, comparative physiological studies were conducted to investigate the phenotypic differences between $y l$ and control (WT and C11) plants. This study improved our understanding of the yellow-green phenotype in birch.

\section{Materials and Methods}

\subsection{Materials}

A birch (Betula platyphylla $\times$ B. pendula) Chl-deficient mutant $y l, B p C C R 1$ overexpression line 11 (C11), and wild type birch (WT) were used as the experimental materials. The yl mutant was derived from $B p C C R 1$ overexpression lines. All the plants were grown in the pots with dimensions of $8 \times 8$ $\mathrm{cm}$ and substrata of $9 \mathrm{~cm}$ under natural conditions and were well watered at the birch breeding base, Harbin, China. Mature leaves from the new stems were collected in the spring. Immediately after harvest, samples were frozen in liquid nitrogen and stored at $-80^{\circ} \mathrm{C}$ for RNA extraction.

\subsection{Methods}

\subsubsection{Measurement of Growth Traits and Pigment Content}

Forty plants of each line (WT, C11, and $y l)$ were used for the measurement of plant height. Each value was the average of the measurements.

Fresh leaves of WT, C11, and $y l$ plants (from first to sixth leaf) were collected during the growing season and used for the measurement of pigment contents, according to the method of Lichtenthaler [21]. The first leaf was the youngest and the sixth leaf was the oldest on the main stem. Chl and carotenoid (Car) were extracted with $80 \%$ acetone at $4{ }^{\circ} \mathrm{C}$ for $24 \mathrm{~h}$ in the dark, and then calculated from the absorbance at $470 \mathrm{~nm}, 646 \mathrm{~nm}$, and $663 \mathrm{~nm}$ in a Vis-UV spectrophotometer (TU-1901, Persee, China). Contents of $\mathrm{Chl} \mathrm{a}(\mathrm{mg} / \mathrm{g}), \mathrm{Chl} \mathrm{b}(\mathrm{mg} / \mathrm{g})$, and Car $(\mathrm{mg} / \mathrm{g})$ were calculated as follows: 


$$
\begin{gathered}
C_{\mathrm{Chl} \mathrm{a}}(\mathrm{mg} / \mathrm{L})=12.21 \mathrm{~A}_{663}-2.81 \mathrm{~A}_{646} \\
C_{\mathrm{Chl} \mathrm{b}}(\mathrm{mg} / \mathrm{L})=20.13 \mathrm{~A}_{646}-5.03 \mathrm{~A}_{663} \\
\mathrm{C}_{\mathrm{Car}}(\mathrm{mg} / \mathrm{L})=4.37 A_{470}+2.11 A_{663}-9.10 A_{646} \\
\mathrm{Chl} \mathrm{a}(\mathrm{mg} / \mathrm{g})=C_{\mathrm{Chl} \mathrm{a}}(\mathrm{mg} / \mathrm{L}) \times V(\mathrm{~L}) / M_{\text {fresh }}(\mathrm{g}) \\
\mathrm{Chl} \mathrm{b}(\mathrm{mg} / \mathrm{g})=C_{\text {Chl b }}(\mathrm{mg} / \mathrm{L}) \times V(\mathrm{~L}) / M_{\text {fresh }}(\mathrm{g}) \\
\operatorname{Car}(\mathrm{mg} / \mathrm{g})=C_{\text {car }}(\mathrm{mg} / \mathrm{L}) \times V(\mathrm{~L}) / M_{\text {fresh }}(\mathrm{g}) \\
\mathrm{Chl} \mathrm{a} / \mathrm{b} \text { ratio }=\mathrm{Chl} \text { a } / \mathrm{Chl} \mathrm{b}
\end{gathered}
$$

\subsubsection{Light Microscopy}

The fourth leaves of WT, C11, and $y l$ were used as samples and fixed in FAA (formaldehyde, glacial acetic acid and 50\% ethyl alcohol, $\mathrm{V}: \mathrm{V}: \mathrm{V}=1: 1: 18$ ) for $24 \mathrm{~h}$, dehydrated in a graded ethanol series and xylene, and then embedded in paraffin wax. Sections $(10 \mu \mathrm{m}$ thick) were stained with safranine and fast green dyes. The cell structures of the samples were examined and photographed using an Olympus DP26 digital camera (Olympus, Tokyo, Japan). Five leaf anatomical features, including lamina thickness (LT), adaxial epidermis thickness (UE), abaxial epidermis thickness (LE), palisade parenchyma thickness (PT), and spongy parenchyma thickness (ST), were examined with the cellSens Entry software. Additionally, the palisade parenchyma/mesophyll ratio was calculated. Four positions of each section were measured and each value of leaf anatomical feature was the average of 20 measurements from five individual plants.

\subsubsection{Leaf Gas-Exchange Measurement}

The net photosynthetic rate (Pn) of the fourth leaves from WT, C11, and $y l$ was measured using an Li-6400 portable photosynthesis system (LI-COR Inc, Lincoln, NE, USA) at 9:00-11:00 am on sunny days. $\mathrm{CO}_{2}$ concentration was controlled at $400 \mu \mathrm{mol} \mathrm{mol}^{-1}$. Relative air humidity was about $50 \%$ and leaf temperature was about $28^{\circ} \mathrm{C}$. The default red/blue LED light source (LI6400-02B) was chosen as the light source. The photosynthetic curves were made against the light intensity of 2000, 1800, $1500,1200,1000,800,600,400,200,100,50,20$, and $0 \mu \mathrm{mol}$ photos $\mathrm{m}^{-2} \mathrm{~s}^{-1}$. About $15 \mathrm{~min}$ light adaptation was applied to leaves at an initial light step before $\mathrm{CO}_{2}$ measurement and then the values were recorded when they were stable at each light step. The measurements of WT, C11, and $y l$ were made under the same conditions (including time interval of illumination). The averaged values of each light step for each plant were used in the light-response curve.

\subsubsection{RNA Extraction, Library Construction, and RNA-seq}

The fourth leaves of WT, C11, and $y l$ were used as samples. Total RNAs were extracted from leaf samples using the CTAB (cetyltrimethylammonium bromide) method [22]. A summary of the procedure was as follows: The samples were individually milled in a mortar with liquid nitrogen and then incubated with $2 \% \mathrm{CTAB}$ (added $2 \% \beta$-mercaptoethanol) at $65^{\circ} \mathrm{C}$ in a water-bath for $5 \mathrm{~min}$. The samples were centrifuged at $13,400 \mathrm{~g}$ for $10 \mathrm{~min}$ and an equal volume of chloroform was added to the supernatant. A half volume of ethyl alcohol and 0.8 times volume of $5 \mathrm{~mol} / \mathrm{L} \mathrm{LiCl}$ were then added to the supernatant after centrifuging at $13,400 \mathrm{~g}, 4{ }^{\circ} \mathrm{C}$ for $10 \mathrm{~min}$. After standing for $10 \mathrm{~min}$, the samples were centrifuged at $13,400 \mathrm{~g}, 4{ }^{\circ} \mathrm{C}$ for $20 \mathrm{~min}$. Then, the precipitates were washed in $70 \%$ ethanol and dried. RNAs were dissolved with diethylpyrocarbonate (DEPC)-treated water and treated with DNaseI. RNA quality, purity, and integrity were detected by $1 \%$ agrose gel electrophoresis, a NanoDrop2000 microvolume spectrophotometer (Thermo, Waltham, MA, USA) and an Agilent 2100 Bioanalyzer (Agilent, Palo Alto, CA, USA), respectively. RNAs from three independent replicates were mixed by equal volume. Poly (A) mRNA was enriched using Oligo (dT)-magnetic beads and cleaved into short fragments with fragmentation buffer. These short fragments were used as templates to 
synthesize the first-strand cDNA. Second-strand cDNA was synthesized using buffer, dNTPs, RNaseH, and DNA polymerase I. Purified cDNA was used as a template for PCR amplification and library construction. Lastly, the library was sequenced on an Illumina HiSeq ${ }^{\mathrm{TM}} 2500$ platform by Biomarker Technology Company (Beijing, China). CTAB, $\beta$-mercaptoethanol, chloroform, ethyl alcohol, LiCl, and DEPC were bought from Sigma-Aldrich, St. Louis, MO, USA. DNaseI, dNTPs, RNaseH, and DNA polymerase I were bought from Promega, Madison, WI, USA.

\subsubsection{Gene Annotation and DEG Analysis}

To elucidate the reason for the different phenotype observed in $y l$, we explored the gene expression of WT, C11, and $y l$ at the molecular level. After removing adapters and low-quality sequences, we generated an average of approximately 3.46 Gb RNA-seq data with 91.28\% Q30 bases and $46.28 \%$ GC content for each sample in the transcriptome sequencing. More than $82.21 \%$ of the clean reads were mapped to the birch reference genome [23] using TopHat2 [24]. Gene functions were annotated using the Nr, Swiss-Prot, Kyoto Encyclopedia of Genes and Genomes (KEGG), Eukaryotic Orthologous Groups (KOG), Clusters of Orthologous Groups (COG) and Gene Ontology (GO) databases. The expression level of each gene was calculated using FPKM (Fragments per kilobase per million mapped reads). Differentially expressed genes (DEGs) between each two-sample comparison were defined with fold change $\geq 2$ and FDR (false discovery rate) $<0.01$ as a threshold, according to the statistical analysis performed by EBSeq. The percentages of DEGs in Go classification were calculated as follows:

$$
\text { Percentages of genes }=(\text { Number DEGs in a specific term }) /(\text { Number all DEGs }) \times 100 \%
$$

KEGG pathway terms with corrected enrichment $p$ values less than 0.05 (Fisher's exact test) were considered to be significantly enriched. The genes involved in photosynthesis-antenna proteins were extracted according to the functional annotation information of the genes.

\subsubsection{RNA Extraction and Quantitative RT-PCR}

Total RNAs were extracted from the functional leaves of the WT, C11, and $y l$ lines as described in 2.1.4 and treated with DNaseI (Promega, Madison, WI, USA). cDNA was synthesized from $1 \mu \mathrm{g}$ RNA of WT, C11, and yl using a ReverTreAce ${ }^{\circledR}$ qPCR RT Kit (Toyobo, Osaka, Japan), according to the manufacturers' instructions, respectively. The procedure was as follows: RNA was incubated with 5x RT Master Mix at $37^{\circ} \mathrm{C}$ for $15 \mathrm{~min}, 50{ }^{\circ} \mathrm{C}$ for $5 \mathrm{~min}$, and $98^{\circ} \mathrm{C}$ for $5 \mathrm{~min}$, and then diluted 10-fold with nuclease-free water. The quantitative (q)RT-PCR was performed on a 7500 real-time PCR system (Applied Biosystems, Darmstadt, Germany) using SYBR ${ }^{\circledR}$ Green PCR master mix (Toyobo, Osaka, Japan). Each qRT-PCR reaction (total $20 \mu \mathrm{L}$ ) contained $2 \mu \mathrm{L}$ of cDNA, $10 \mu \mathrm{L}$ of $2 \times$ SYBR Green Real-time PCR Master mix, $0.5 \mu \mathrm{L}$ of each PCR primer, and $7 \mu \mathrm{L}$ of nuclease-free water. qRT-PCR conditions were as follows: $95^{\circ} \mathrm{C}$ for $30 \mathrm{~s}$ followed by 45 cycles of $95{ }^{\circ} \mathrm{C}$ for $15 \mathrm{~s}, 60{ }^{\circ} \mathrm{C}$ for $45 \mathrm{~s}$, and a final extension at $72{ }^{\circ} \mathrm{C}$ for $30 \mathrm{~s}$. The results were calculated using the $2^{-\Delta \Delta \mathrm{Ct}}$ method [25], and $B p 18 \mathrm{~S}$ $r R N A$ was selected as an internal control gene [26]. Primer sequences are listed in Table 1.

\subsubsection{Statistical Analysis}

Data were analyzed using SPSS statistics software, version 19.0 (International Business Machines, Armonk, NY, USA). Differences between the means of each line on leaf anatomical and plant height were determined using one-way analysis of variance and the Duncan multiple comparison procedure. A $p$ value less than 0.05 was considered statistically significant and labeled with a different letter. The same letter represented that they were not significantly different. The correlations between pigment contents and Pn, stomatal conductance (Gs) and Pn, Gs and transpiration rate (Tr), and $\mathrm{Tr}$ and Pn were evaluated using Pearson's correlation coefficients. The relationship between Tr and Pn was tested for linear, exponential, and logarithmic functions and the best fit regressions were selected. 
Correlation coefficients $\left(R^{2}\right)$ and equations were obtained from nonlinear regression analysis of $\mathrm{Tr}$ and Pn using Origin Pro software, version 8.1 (OriginLab, Northampton, MA, USA).

Table 1. The primer sequences used in qRT-PCR.

\begin{tabular}{ccc}
\hline Gene & Forward Primer $\left(\mathbf{5}^{\prime} \mathbf{- 3}^{\prime} \mathbf{)}\right.$ & Reverse Primer $\mathbf{( 5}^{\prime} \mathbf{- 3}^{\prime} \mathbf{)}$ \\
\hline Bpev01.c0080.g0006.m0001 & GGCTCAATCCAGCATGGTTGC & CCACACCTGCATGCATTGCAC \\
Bpev01.c0577.g0002.m0001 & CGTTCATGGTGGACCTGAGCC & AGCTAAAGTGAGGGACTTTGTCGAT \\
Bpev01.c0167.g0013.m0001 & CACAACATAGCCAGCCACCTTC & GTCGGTGCTACCCAAGGACTC \\
Bpev01.c0243.g0056.m0001 & CAACGGACGTTATGCCATGTTGG & TCCCAGCCGGCGGAATTAC \\
Bpev01.c0264.g0036.m0001 & CGGTGAGGCCGTGTGGTT & CGCCCATCAGGATGACCTGT \\
Bpev01.c1040.g0049.m0001 & CCCGAGACATTTGCCAAGAACC & GCCTTGAACCAGACTGCCTCTC \\
Bpev01.c0190.g0044.m0001 & CTTGGCCGGCGATGTGATC & GCCAACATGGCCCACCTC \\
Bpev01.c0621.g0012.m0001 & GCAATAGGCCTTGCCTCCTTCATAG & CGAGTACCCGTCTTCTCATTCGC \\
Bpev01.c0984.g0005.m0001 & GGGAGACTAAGGTACAAGCAGTGG & CCAGCTGCTCAATTGCTTCAGAG \\
Bpev01.c0401.g0006.m0001 & GGGAAGGCAAGGCTAGTGCAG & GCCTTCAACAGCAAGGCAAGT \\
Bpev01.c0894.g0003.m0001 & CCTCCAACAGGGAGTGGCAAC & CTGTCAATCATCCCAGAACAGCTTG \\
Bpev01.c1891.g0003.m0001 & GGCGTTCCGTATATGAGCCTCTTC & GGCTACTGCTGTTTTACCGGTCT \\
\hline
\end{tabular}

\section{Results}

\subsection{Pigment Contents and Leaf Anatomical Structure of Chl-Deficient Mutant yl}

Previously, we transformed the BpCCR1 gene to a hybrid birch, WT (Betula platyphylla $\times$ B. Pendula) by Agrobacterium tumefaciens. We obtained 19 BpCCR1-overexpressing transgenic lines. Among them, a transgenic line $(y l)$ displayed a yellow-green leaf phenotype, which was distinct from other transgenic lines, including C11. During the growth season, the $y l$ mutant exhibited yellow-green leaves, while C11 and WT exhibited green leaves (Figure 1a,b). In order to investigate the difference, we measured the pigment contents and leaf anatomical structure of $y l$. We measured the $\mathrm{Chl}$ and Car contents from the first leaf to the sixth leaf. The result showed that the pigment contents, including Chl a, Chl b, and Car, were increased from the first leaf to sixth leaf in all samples (Figure 1c). However, all pigment levels were lower in the leaves of $y l$ than those of WT and C11. Chl a in $y l$ was decreased by 44-65\% of WT, and $41-57 \%$ of C11. Chl b in $y l$ was decreased by $62-79 \%$ of WT, and $61-73 \%$ of C11. Similarly, Car in $y l$ was decreased by $40-55 \%$ compared with WT, and 30-54\% compared with C11. The ratio of $\mathrm{Chl}$ a to $\mathrm{Chl} b$ was increased in all leaves of $y l$.

We then investigated the leaf anatomical structure of WT, C11, and $y l$, as leaves are important organs for photosynthesis, and their anatomical structures could affect photosynthetic and physiological activities. A significant reduction in the lamina thickness of $y l$ leaves compared with the leaves of WT and C11 was observed in transverse sections of the leaf blades (Figure 2a). Also, adaxial epidermis, abaxial epidermis, palisade parenchyma, and spongy parenchyma were reduced in thickness in $y l$ leaves (Figure $2 b$ ). However, there was no difference in the ratio of palisade to spongy parenchyma.

\subsection{Changes in Photosynthesis and Growth}

To examine the effect of low pigment contents on photosynthesis in the $y l$ mutant, we measured the photosynthetic rate of WT, C11, and $y l$. The result showed that $y l$ had a lower net photosynthetic rate and a lower transpiration rate than WT and C11 at all light intensities determined (Figure 3a,b).

We then investigated the correlations between pigment contents, transpiration rate, stomatal conductance, and Pn. The results showed that the Pn values had a strong relationship with total $\mathrm{Chl}, \mathrm{Chl} \mathrm{a}, \mathrm{Chl} \mathrm{b}$, and Car under high light intensity $(p<0.05)$. The correlations between pigment contents and Pn were decreased under low light intensity, revealing that not all pigments participated in photosynthesis under low light intensity (Table S1). In addition, we found that Pn and Tr values displayed a significant positive correlation $(p<0.01)$ with Gs in WT, C11, and yl plants. Similarly, there was also a highly significant correlation $(p<0.01)$ between Pn and Tr in WT, C11, and yl plants (Table 2). 
Then, we analyzed the scatter plots between Pn and Tr and found that the exponential decay function was the best fit. The curve could be divided into two stages. In the first stage, the Pn value increased with the rising Tr and the curve was likely to be linear. Gs may be the primary limiting factor in this stage. In the second stage, Pn increased slowly or mantained invariability along with the increase of Tr. Gs may not be the primary limiting factor in this stage (Figure $4 a-c$ ).

To explore the effect of low photosynthesis on growth, we measured the height of WT, C11, and $y l$ plants. The heights of one-year-old WT and C11 were $36.7 \mathrm{~cm}$ and $37.0 \mathrm{~cm}$, while the $y l \mathrm{mutant}$ was $30.9 \mathrm{~cm}$, about $84 \%$ and $83 \%$ of WT and C11, respectively. The result revealed that $y l$ grew slower compared to WT and C11 (Figure 3c).

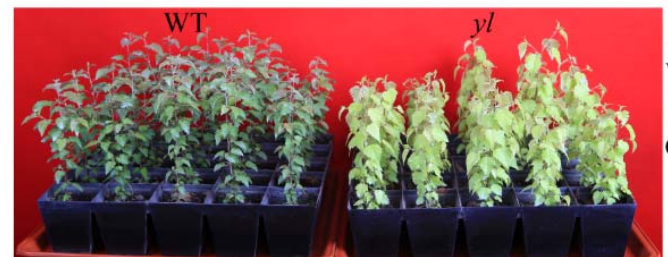

(a)
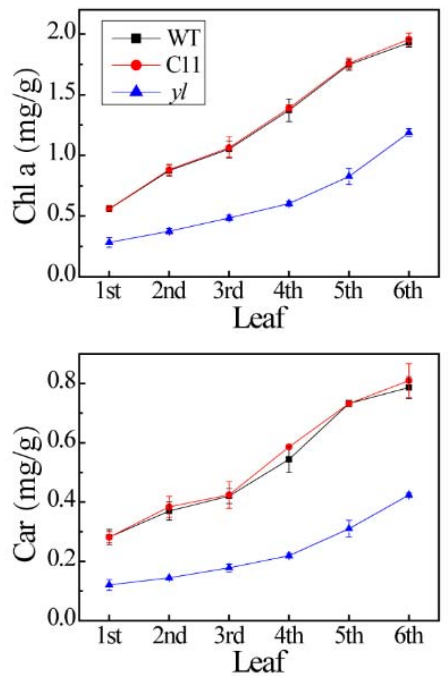

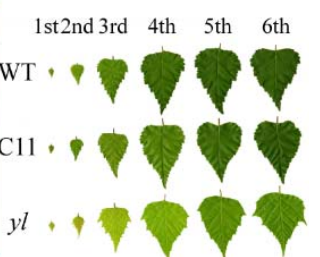

(b)
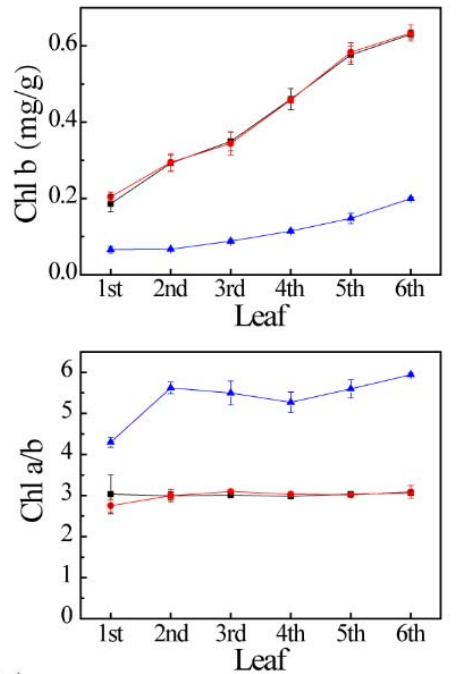

(c)

Figure 1. Growth performance and pigment content of wild type birch (WT), BpCCR1 overexpression line 11 (C11) and yellow-green leaf mutant (yl) lines. (a) Growth performance of one-year-old WT and yl plants. (b) The leaves from first to sixth of WT, C11, and yl lines. (c) Chl a, Chl b, Car, and Chl a/b in first to sixth leaves of WT, C11, and $y l$ lines. Error bars represent the standard deviation (SD) of three independent experiments. 
WT

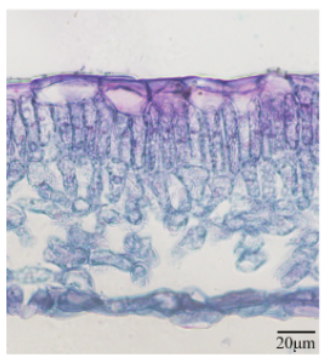

C11

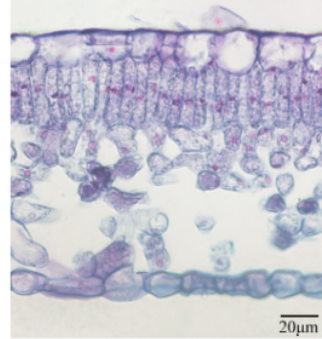

$y l$

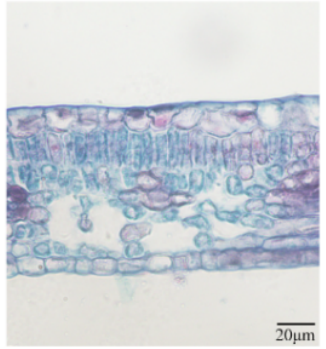

(a)

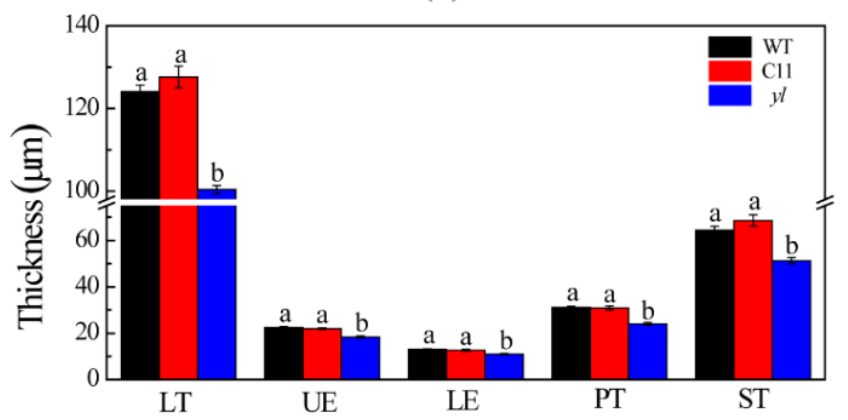

(b)

Figure 2. Leaf anatomical characteristics of WT, C11, and $y l$. (a) Leaf transections of WT, C11, and $y l$ lines. (b) Leaf anatomical structure of WT, C11, and yl. Lamina thickness, LT. Adaxial epidermis thickness, UE. Abaxial epidermis thickness, LE. Palisade parenchyma thickness, PT. Spongy parenchyma thickness, ST. Error bars represent the standard deviation (SD) of 20 measurements from five individual plants.

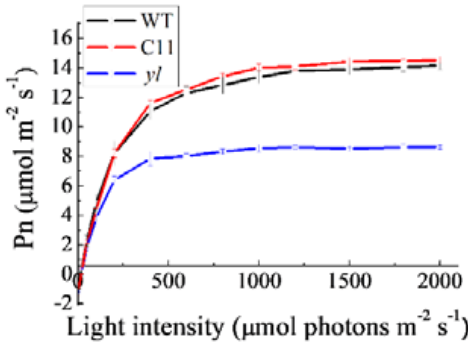

(a)

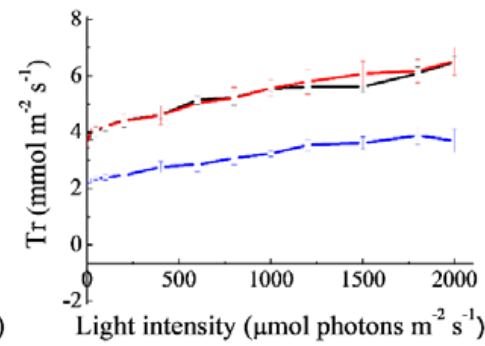

(b)

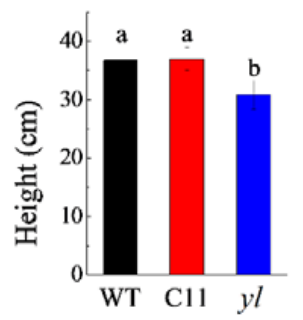

(c)

Figure 3. Photosynthetic and growth performance of WT, C11, and $y l$ lines. Photosynthetic rate (a) and transpiration rate (b) of WT, C11, and $y l$. Error bars represent the SD of three measurements. (c) Plant height of WT, C11, and $y l$. Error bars represent the SD of 40 measurements.

Table 2. The correlation analysis of transpiration rate (Tr), stomatal conductance (Gs), and Pn in WT, C11, and $y l .{ }^{* *}$ Correlation is significant at the 0.01 level (2-tailed).

\begin{tabular}{|c|c|c|c|c|c|c|}
\hline Correlation & $\begin{array}{c}\text { Gs in WT } \\
\left(\mathrm{mol} \mathrm{m}^{-2} \mathrm{~s}^{-1}\right)\end{array}$ & $\begin{array}{c}\text { Tr in WT } \\
\left(\mathrm{mmol} \mathrm{m}^{-2} \mathrm{~s}^{-1}\right)\end{array}$ & $\begin{array}{c}\text { Gs in C11 } \\
\left(\mathrm{mol} \mathrm{m}^{-2} \mathrm{~s}^{-1}\right)\end{array}$ & $\begin{array}{c}\operatorname{Tr} \text { in } \mathrm{C} 11 \\
\left(\mathrm{mmol} \mathrm{m}^{-2} \mathrm{~s}^{-1}\right)\end{array}$ & $\begin{array}{c}\text { Gs in } y l \\
\left(\mathrm{~mol} \mathrm{~m}^{-2} \mathrm{~s}^{-1}\right)\end{array}$ & $\begin{array}{c}\operatorname{Tr} \text { in } y l \\
\left(\mathrm{mmol} \mathrm{m}^{-2} \mathrm{~s}^{-1}\right)\end{array}$ \\
\hline $\begin{array}{c}\text { Tr in WT } \\
\left(\mathrm{mmol} \mathrm{m}^{-2} \mathrm{~s}^{-1}\right)\end{array}$ & $0.987^{* *}$ & - & - & - & - & - \\
\hline $\begin{array}{c}\text { Pn in WT } \\
\left(\mu \mathrm{mol} \mathrm{m}^{-2} \mathrm{~s}^{-1}\right)\end{array}$ & 0.860 ** & $0.915^{* *}$ & - & - & - & - \\
\hline $\begin{array}{c}\text { Tr in C11 } \\
\left(\mathrm{mmol} \mathrm{m}^{-2} \mathrm{~s}^{-1}\right)\end{array}$ & - & - & $0.986 * *$ & - & - & - \\
\hline $\begin{array}{c}\text { Pn in C11 } \\
\left(\mu \mathrm{mol} \mathrm{m}{ }^{-2} \mathrm{~s}^{-1}\right)\end{array}$ & - & - & $0.856^{* *}$ & $0.895^{* *}$ & - & - \\
\hline $\begin{array}{c}\operatorname{Tr} \text { in } y l \\
\left(\mathrm{mmol} \mathrm{m}^{-2} \mathrm{~s}^{-1}\right)\end{array}$ & - & - & - & - & $0.991 * *$ & - \\
\hline $\begin{array}{c}\text { Pn in } y l \\
\left(\mu \mathrm{mol} \mathrm{m}^{-2} \mathrm{~s}^{-1}\right)\end{array}$ & - & - & - & - & $0.773^{* *}$ & $0.815^{* *}$ \\
\hline
\end{tabular}




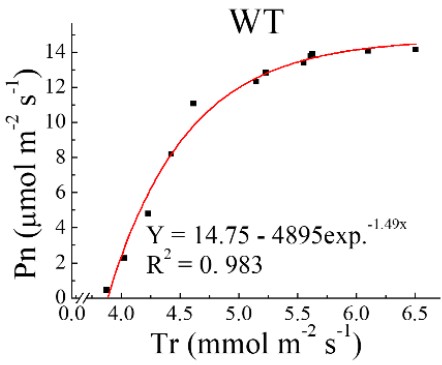

(a)

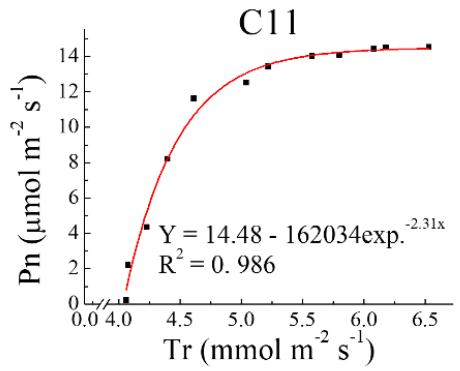

(b)

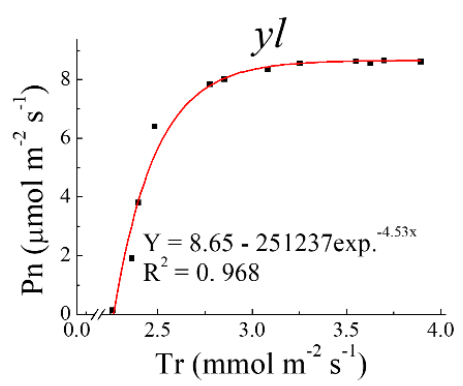

(c)

Figure 4. The scatter plots between Pn and Tr in WT, C11, and $y l$.

\subsection{Differently Expressed Genes between yl and Control Plants}

In order to generate DEGs, two transcriptome comparisons were carried-out, including C11 vs. yl and WT vs. $y l$. As a result, 1163 genes (874 down-regulated and 289 up-regulated) were found to be differentially expressed in $y l$ compared with C11. 930 genes, including 755 down-regulated and 175 up-regulated that were differentially expressed in $y l$ compared with WT (Figure 5a).

GO categories were then assigned to evaluate the potential functions of these DEGs according to the biological process, cellular component, and molecular function ontology. In the biological process, the DEGs were classified into twenty categories, and the most three overrepresented terms were cellular process, single-organism process, and metabolic process. For cell components, there are many DEGs involved in cells, cell parts, and organelles. For molecular functions, most of the DEGs were participates in binding and catalytic activity, as shown in Figure $5 \mathrm{~b}$.

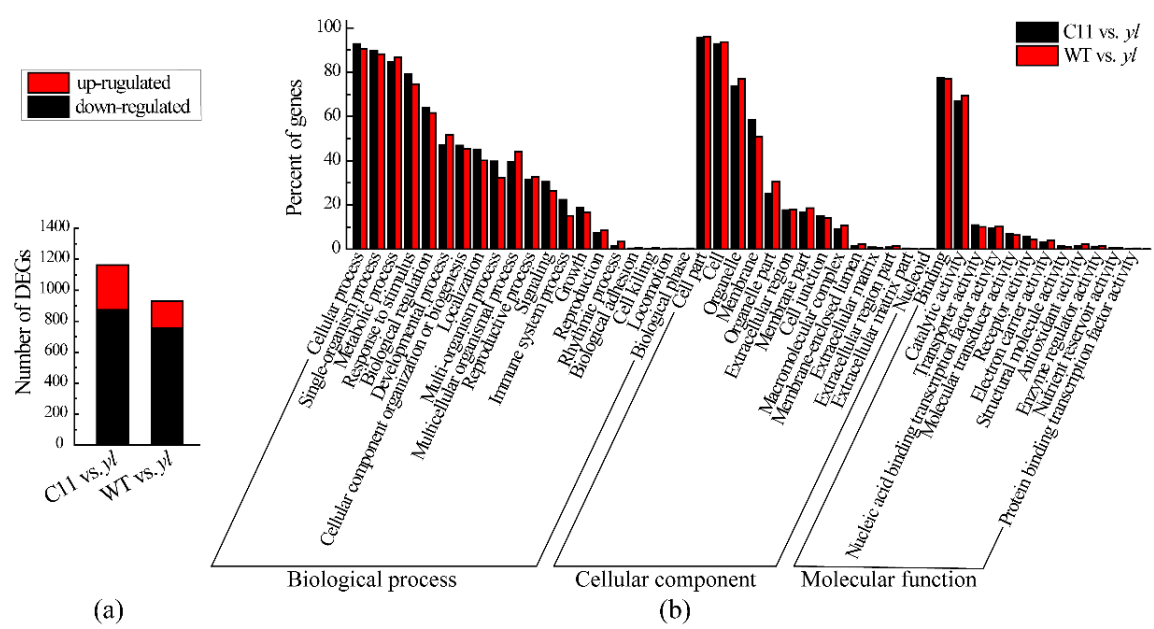

Figure 5. Differentially expressed genes (DEGs) analysis based on Gene ontology. (a) Number of DEGs in C11 vs. $y l$ and WT vs. $y l$. (b) Gene ontology classification of DEGs in C11 vs. $y l$ and WT vs. $y l$.

\subsection{KEGG Pathway Analysis of DEGs}

The DEGs were further subjected to KEGG pathway analysis to identify the enriched biological pathways. The DEGs of C11 vs. $y l$ and WT vs. $y l$ were mapped to 81 and 85 KEGG pathways, respectively. Among them, two pathways, including photosynthesis-antenna proteins and phenylalanine metabolism, were considered significantly enriched at a cut-off P-value $<0.05$ and FDR $<0.05$ in both C11 vs. $y l$ and WT vs. $y l$. Photosynthesis-antenna proteins represented the most significantly enriched pathway in the DEGs of $y l$ compared to WT and C11 (Figure 6a,b). 
C11 vs. $y l$

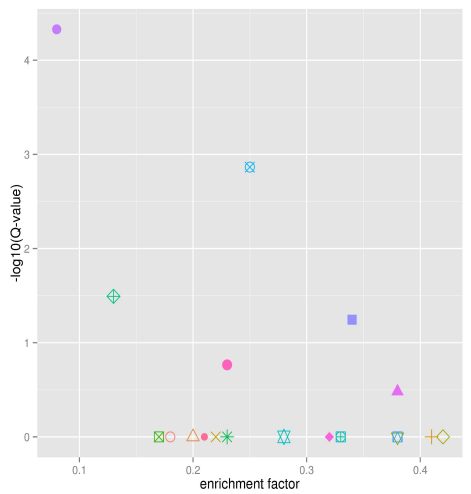

(a)
WT vs. $y l$
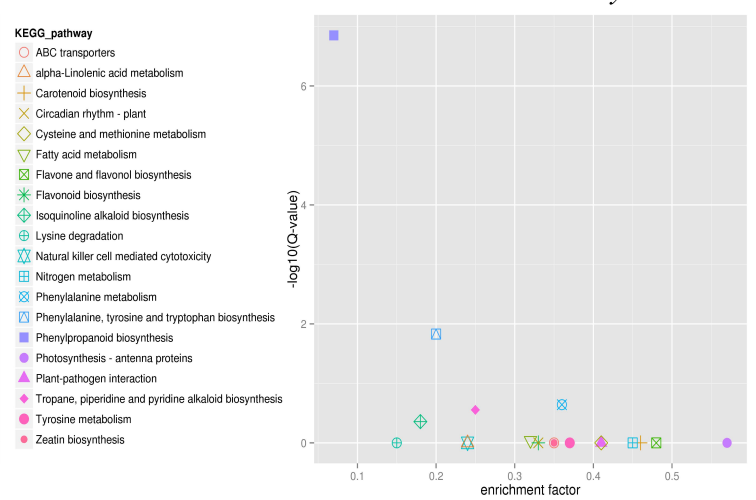

(b)

Figure 6. KEGG-based pathway enrichment of DEGs in C11 vs. $y l$ and WT vs. $y l$.

We then explored the photosynthesis-antenna proteins pathway in more detail. A total of twenty-one Lhc (light-harvesting complex) genes were found to be related to the antenna proteins in the birch genome. However, seven of these genes were differently expressed in $y l$ compared to control plants and six displayed low or undetectable levels of expression in all samples. One gene (Bpev01.c0243.g0056.m0001) involved in light-harvesting the $\mathrm{Chl} \mathrm{a/b}$ binding protein Lhca3 of Photosystem I, three genes (Bpev01.c0362.g0012.m0001, Bpev01.c0264.g0036.m0001, Bpev01.c1767.g0010.m0001) involved in light-harvesting the $\mathrm{Chl} \mathrm{a/b}$ binding protein Lhcb1, and one gene (Bpev01.c1040.g0049.m0001) involved in light-harvesting the $\mathrm{Chl} \mathrm{a} / \mathrm{b}$ binding protein Lhcb2 of Photosystem II showed significantly reduced transcript levels in $y l$. Two genes (Bpev01.c0190.g0044.m0001, Bpev01.c0841.g0007.m0001) were involved in light-harvesting the $\mathrm{Chl} \mathrm{a/b}$ binding protein Lhcb4 of Photosystem II, and Bpev01.c0190.g0044.m0001 was down-regulated and Bpev01.c0841.g0007.m0001 was up-regulated (Figure 7a,b). These results suggested that the changes in the photosynthesis-antenna proteins pathway were important to the unique phenotype of $y l$.

Light-harvesting chlorophyll protein complex

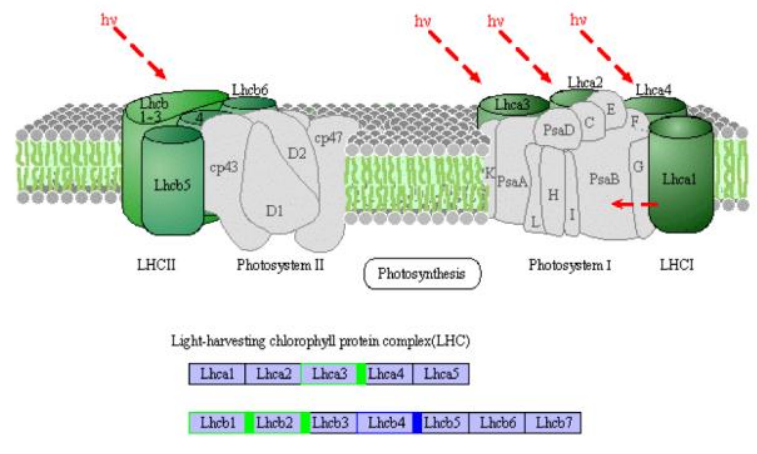

(a)

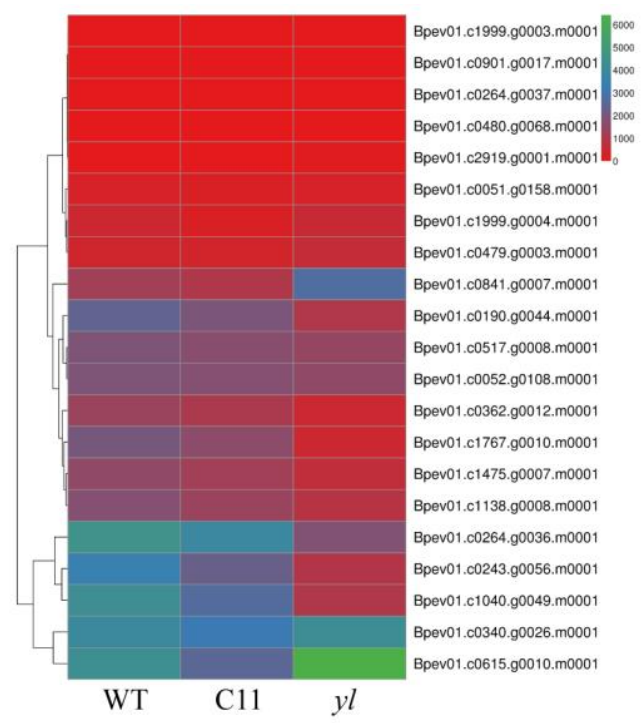

(b)

Figure 7. Expression pattern of genes involved in light-harvesting Chl complex in WT, C11, and $y l$. (a) DEGs in the pathway of light-harvesting Chl complex. Green box represents down-regulated genes. Blue box represents down-regulated and up-regulated genes. (b) Expression of all genes involved in light-harvesting Chl complex in WT, C11, and $y l$. 


\section{5. qRT-PCR Verification of RNA-seq}

To test the reliability of RNA-Seq, we selected 12 functionally important and representative genes for validation using qRT-PCR, including two non-DEGs, five down-regulated genes, and five up-regulated genes (Table 3). The expression of all these genes obtained via qRT-PCR showed a similar pattern to that detected by transcriptome sequencing (Figure 8).

Table 3. The genes used for qRT-PCR.

\begin{tabular}{cc}
\hline Gene & C11 and WT vs. $y \boldsymbol{l}$ \\
\hline Bpev01.c0080.g0006.m0001 & normal \\
Bpev01.c0577.g0002.m0001 & normal \\
Bpev01.c0167.g0013.m0001 & down-regulated \\
Bpev01.c0243.g0056.m0001 & down-regulated \\
Bpev01.c0264.g0036.m0001 & down-regulated \\
Bpev01.c1040.g0049.m0001 & down-regulated \\
Bpev01.c0190.g0044.m0001 & down-regulated \\
Bpev01.c0621.g0012.m0001 & up-regulated \\
Bpev01.c0984.g0005.m0001 & up-regulated \\
Bpev01.c0401.g0006.m0001 & up-regulated \\
Bpev01.c0894.g0003.m0001 & up-regulated \\
Bpev01.c1891.g0003.m0001 & up-regulated \\
\hline
\end{tabular}
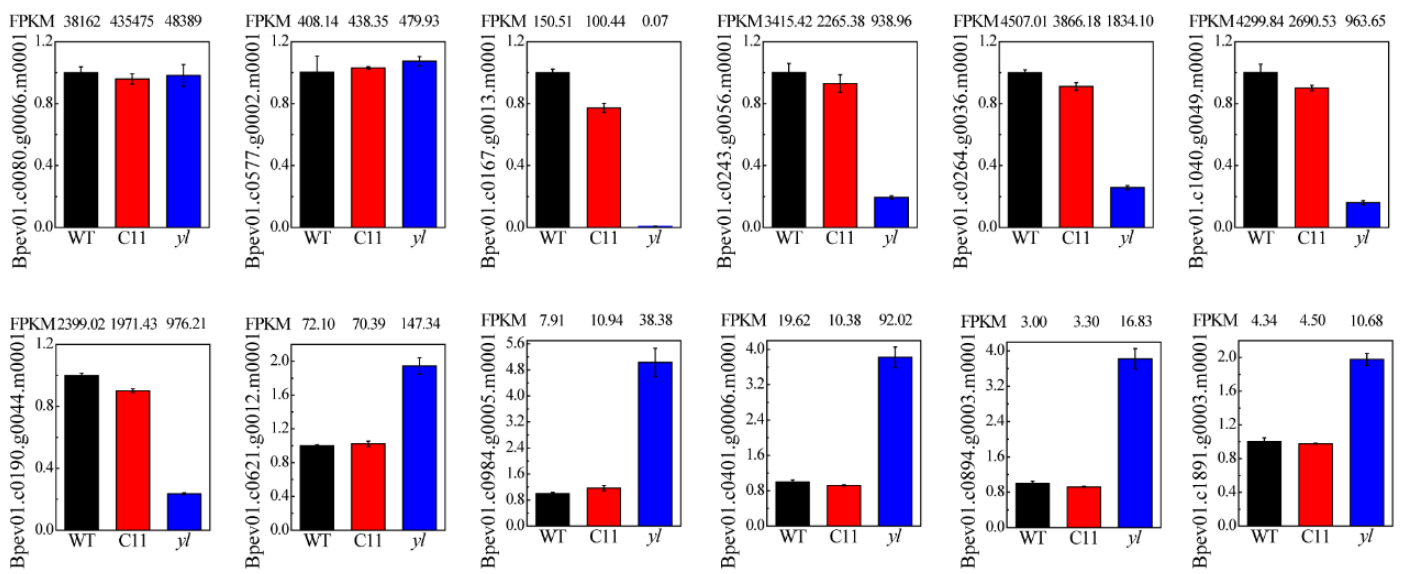

Figure 8. Quantificational real-time PCR verification of RNA-seq. Error bars represent the SD of three measurements.

\section{Discussion}

In this study, we reported a Chl-deficient mutant $y l$ that produced yellow-green leaves in birch. The mutant was isolated from BpCCR1 transgenic lines in birch plant breeding. Physiological analysis and gene expression characterization of $y l$ were performed to investigate the difference between $y l$ and control plants (WT and C11).

$\mathrm{Chl}$ and Car are the main pigments that trap light energy in leaf tissue. It has been demonstrated that the leaves of Chl-deficient mutants always contain less Chl and Car. Some mutants also showed a change in the ratio of $\mathrm{Chl} \mathrm{a/b}[16,27,28]$. Consistent with the previous research, pigment analysis of $y l$ showed that $\mathrm{Chl}$ a, $\mathrm{Chl} \mathrm{b}$, and Car were reduced in young leaf or mature leaf specimens, compared to WT and C11. We also found that the ratio of $\mathrm{Chl} \mathrm{a/b}$ was increased in the $y l$ plants (Figure 1 ). $\mathrm{Chl} \mathrm{b}$ is thought to be essential to the stability of the light-harvesting $\mathrm{Chl} \mathrm{a} / \mathrm{b}$ protein complex [29]. Thus, the decreased $\mathrm{Chl}$ content and increased $\mathrm{Chl} \mathrm{a/b}$ ratio in $y l$ indicated that there might be fewer light-harvesting antenna complexes than in the WT and C11 controls. There are numerous Chl-deficient mutants that have shown reduced amounts of light-harvesting proteins (LHC) in the thylakoid membranes of the chloroplast [30,31]. Andersson reported that the absence of Lhcb1 and 
Lhcb2 proteins in Arabidopsis showed reduced Chl levels and increased Chl a/b [32]. According to transcriptomic analysis, DEGs of $y l$ compared with WT and C11 were both significantly enriched in photosynthesis-antenna proteins (Figures 6 and 7). These results revealed that the expression change of genes involved in photosynthesis-antenna proteins plays an important role in the formation of the yellow-green leaf phenotype in $y l$.

Green plants absorb light energy to convert $\mathrm{CO}_{2}$ and water into carbohydrates and oxygen through photosynthesis. Photosynthesis is the key process that provides energy for catabolic processes and growth in plants. It has been reported that photosynthesis could be influenced by many environmental factors, such as intensity, spectrum and duration of illumination, mechanical wounding, and heating $[33,34]$. These stimulations would induce the generation and propagation of variation potential [35]. Then, electron flow connected to $\mathrm{pH}$ would be changed and the light-harvesting complex would transfer to photosystem II (PSII). As a result, photosynthesis would be decreased in plants [36]. Photosynthesis is a complex process. The changes in pigment contents, stomata conductance, and gene expressions may also affect photosynthesis [37]. Due to the reduced pigment contents, most chlorina mutants have a poorer photosynthetic performance than that observed in wild type specimens $[9,38]$. However, this is not true for all chlorine mutants. For example, a chlorina rice mutant Huangyu B was found to have a higher photosynthetic efficiency than its wild type [39], and the photosynthetic rate of a Chl-deficient mutant siygl1 in foxtail millet was even higher than that of Yugu1 plants during the reproductive growth stage [15]. In this study, $y l$ showed a reduced pigment, including Chl a, Chl b, and Car contents (Figure 1). The photosynthetic rate, stomata conductance, and transpiration rate of the $y l$ line were lower than WT and C11 under all light intensities set (Figure 3). Additionally, the measurement of photosynthesis in WT, C11, and $y l$ was performed under the same conditions. We also found that there was a significant positive correlation between stomata conductance, pigment contents, and photosynthetic rate. RNA-seq results showed that many genes related to photosynthesis-antenna proteins were down-regulated in $y l$ compared to WT and C11 (Figure 7). As a result, the energy absorbed, trapped, and transferred in photosystem I (PSI) and PSII would probably be affected. Taken together, the decreased photosynthesis in $y l$ was probably mainly due to the low expression level of antenna protein genes, and reduced photosynthetic pigment contents. The difference in Pn may underlie the retarded growth in one-year-old yl mutant plants (Figures 1 and 3).

The molecular mechanism of leaf color mutation is complex. Mutation of genes related to chloroplast development, blocked in photosynthetic pigment biosynthesis, blocked in chloroplast protein transport, and blocked in phytochrome regulation would lead to the formation of a yellow-green mutant [26-31]. Studies have shown that plentiful genes are related to the yellow-green leaf phenotype in plants. Examples are cytokinin-responsive gata transcription factor1, Cga1 [40]; chaperone protein $\mathrm{ClpC}, \mathrm{ClpC1}$ [41]; signal recognition particle $43 \mathrm{kDa}$ protein, cpSRP43 [42]; chloroplast Signal recognition particle subunit, cpSRP54 [43]; metallo-beta-lactamase, GRY79 [44]; nuclear transcription factor Y, HAP3A [45]; NADPH-dependent thioredoxin reductase C, NTRC [46]; protein stay-green, SGR [47]; and YbeY endoribonuclease, YbeY [38]. However, the expression of all these genes did not change in the $y l$ transcriptome (Table 4 ).

Table 4. The expression of well-known genes related to leaf color in the transcriptional level of a yl mutant.

\begin{tabular}{ccccc}
\hline Gene & ID & Annotation & C11 and WT vs. $y \boldsymbol{l}$ Mutant phenotype \\
\hline Cga1 & Bpev01.c0051.g0036.m0001 & Cytokinin-responsive gata transcription factor1 & normal & yellow leaf \\
ClpC1 & Bpev01.c1202.g0038.m0001 & Chaperone protein ClpC & normal & chlorotic leaf \\
cpSRP43 & Bpev01.c1171.g0012.m0001 & Signal recognition particle 43 kDa protein & normal & yellow-green leaf \\
cpSRP54 & Bpev01.c1238.g0002.m0001 & Chloroplast signal recognition particle subunit & nellow-green leaf \\
GRY79 & Bpev01.c0787.g0006.m0001 & Metallo-beta-lactamase & normal & yellow turn green \\
HAP3A & Bpev01.c0288.g0025.m0001 & Nuclear transcription factor Y & normal & pale-green leaf \\
NTRC & Bpev01.c0029.g0129.m0001 & NADPH-dependent thioredoxin reductase C & normal & light-green leaf \\
SGR & Bpev01.c0717.g0015.m0001 & Protein stay-green & normal & normal \\
YbeY & Bpev01.c1489.g0003.m0001 & YbeY endoribonuclease & pale-green leaf \\
\hline
\end{tabular}


Plastid-to-nucleus retrograde signaling is considered to coordinate nuclear gene expression. Nott has summarized three independent retrograde signaling pathways from previous studies, including signals generated by Mg-Protoporphyrin IX (Mg-Proto IX), chloroplast gene expression, and the redox state of photosynthetic electron transport components [48]. One possibly important function of retrograde signaling is to regulate the biosynthesis of $\mathrm{Chl}$ with the expression of genes for nuclear-encoded Chl-binding proteins, such as the Lhca and Lhcb proteins. Here, we found that the transcription of Chl-binding protein genes ( $L h c$ gene family) was down-regulated via RNA-seq and qRT-PCR analysis (Figures 7 and 8). Members of the Golden2-like (GLK) gene family have been reported to regulate chloroplast development in diverse plant species $[49,50]$. GLK genes are sensitive to retrograde signaling from the chloroplast, and they could then operate downstream of genes for plastid retrograde signaling [51]. The expression of GLK (Bp023762) was only $0.7 \%$ of WT and $0.9 \%$ of C11 (Figure 8). In addition, the PSRP1 gene (BP012524), an encoding ribosomal-binding factor (plastid-specific ribosomal protein 1) that inhibits plastid translation by blocking tRNA-binding sites on ribosomes, was upregulated in the $y l$ mutant. These results suggest that plastid-to-nucleus retrograde signaling triggered in $y l$ may regulate nuclear gene expression.

\section{Conclusions}

In this study, about 1163 DEGs and 930 DEGs were obtained in yl compared with WT and C11, respectively. The DEGs related to the photosynthesis antenna proteins pathway were significantly enriched. In addition, the physiological characteristics analysis showed that the yellow-green leaf mutant $y l$ had reduced amounts of $\mathrm{Chl}$, an increased $\mathrm{Chl} \mathrm{a} / \mathrm{b}$ value, and reduced leaf anatomical compared to control plants. Based on these results, we can conclude that the expression of genes involved in the photosynthesis antenna proteins pathway might be responsible for the lower pigment contents and ratio of $\mathrm{Chl} \mathrm{a} / \mathrm{b}$, resulting in yellow-green leaves in $\mathrm{yl}$.

Supplementary Materials: The following are available online at http:/ /www.mdpi.com/1999-4907/10/2/120/s1, Table S1: The correlation analysis between pigment contents and Pn.

Author Contributions: J.J. and S.C. designed the experiments; H.G. performed the experiments; S.C. analyzed the data; H.G. wrote the manuscript; J.J., G.L., and S.C. revised the manuscript.

Funding: This research was funded by the National Natural Science Foundation of China (No. 31570647) and the 111 Project (B16010).

Acknowledgments: We are grateful to the transgenic material yl generated by Rui Wei and Wenbo Zhang.

Conflicts of Interest: The authors declare no conflict of interest.

\section{References}

1. Zyryanova, O.A.; Terazawa, M.; Koike, T.; Zyryanov, V.I. White birch trees as resource species of Russia: Their distribution, ecophysiological features, multiple utilizations. Eurasian J. For. Res. 2010, 13, $25-40$.

2. Dulamsuren, C.; Hauck, M.; Bader, M.K.-F.; Osokhjargal, D.; Oyungerel, S.; Nyambayar, S.; Runge, M.; Leuschner, C. Water relations and photosynthetic performance in Larix sibirica growing in the forest-steppe ecotone of northern Mongolia. Tree Physiol. 2008, 29, 99-110. [CrossRef]

3. Bekhta, P.; Hiziroglu, S.; Shepelyuk, O. Properties of plywood manufactured from compressed veneer as building material. Mater. Des. 2009, 30,947-953. [CrossRef]

4. Liu, X.; Wang, Q.; Chen, P.; Song, F.; Guan, M.; Jin, L.; Wang, Y.; Yang, C. Four Novel Cellulose Synthase (CESA) Genes from Birch (Betula platyphylla Suk.) Involved in Primary and Secondary Cell Wall Biosynthesis. IJMS 2012, 13, 12195-12212. [CrossRef] [PubMed]

5. Borrega, M.; Tolonen, L.K.; Bardot, F.; Testova, L.; Sixta, H. Potential of hot water extraction of birch wood to produce high-purity dissolving pulp after alkaline pulping. Bioresour. Technol. 2013, 135, 665-671. [CrossRef] [PubMed]

6. Rubert-Nason, K.F.; Holeski, L.M.; Couture, J.J.; Gusse, A.; Undersander, D.J.; Lindroth, R.L. Rapid phytochemical analysis of birch (Betula) and poplar (Populus) foliage by near-infrared reflectance spectroscopy. Anal. Bioanal. Chem. 2012, 405, 1333-1344. [CrossRef] [PubMed] 
7. Lin, L.; Mu, H.; Jiang, J.; Liu, G. Transcriptomic analysis of purple leaf determination in birch. Gene 2013, 526, 251-258. [CrossRef] [PubMed]

8. Zhang, W.B.; Wei, R.; Chen, S.; Jiang, J.; Li, H.Y.; Huang, H.J.; Yang, G.; Wang, S.; Wei, H.R.; Liu, G.F. Functional characterization of CCR in birch (Betula platyphylla $x$ Betula pendula) through overexpression and suppression analysis. Physiol. Plant. 2015, 154, 283-296. [CrossRef]

9. Córdoba, J.; Molina-Cano, J.-L.; Martínez-Carrasco, R.; Morcuende, R.; Pérez, P. Functional and transcriptional characterization of a barley mutant with impaired photosynthesis. Plant Sci. 2016, 244, 19-30. [CrossRef]

10. Liu, J.; Wang, J.; Yao, X.; Dong, X.; Chen, W.; Li, Z.K. Fine mapping and photosynthetic characteristics of the lower chlorophyllb1 mutant in rice (Oryza sativa L.). Plant Breed. 2015, 134, 661-667. [CrossRef]

11. Zhong, X.M.; Sun, S.F.; Li, F.H.; Wang, J.; Shi, Z.S. Photosynthesis of a yellow-green mutant line in maize. Photosynthetica 2015, 53, 499-505. [CrossRef]

12. Li, N.; Jia, J.; Xia, C.; Liu, X.; Kong, X. Characterization and mapping of novel chlorophyll deficient mutant genes in durum wheat. Breed. Sci. 2013, 63, 169-175. [CrossRef] [PubMed]

13. Karaca, M.; Saha, S.; Callahan, F.E.; Jenkins, J.N.; Read, J.J.; Percy, R.G. Molecular and cytological characterization of a cytoplasmic-specific mutant in pima cotton (Gossypium barbadense L.). Euphytica 2004, 139, 187-197. [CrossRef]

14. Wang, L.; Yue, C.; Cao, H.; Zhou, Y.; Zeng, J.; Yang, Y.; Wang, X. Biochemical and transcriptome analyses of a novel chlorophyll-deficient chlorina tea plant cultivar. BMC Plant Biol. 2014, 14, 239. [CrossRef]

15. Li, W.; Tang, S.; Zhang, S.; Shan, J.; Tang, C.; Chen, Q.; Jia, G.; Han, Y.; Zhi, H.; Diao, X. Gene mapping and functional analysis of the novel leaf color gene SiYGL1 in foxtail millet [Setaria italica (L.) P. Beauv]. Physiol. Plant. 2016, 157, 24-37. [CrossRef] [PubMed]

16. Zhu, X.; Guo, S.; Wang, Z.; Du, Q.; Xing, Y.; Zhang, T.; Shen, W.; Sang, X.; Ling, Y.; He, G. Map-based cloning and functional analysis of YGL8, which controls leaf colour in rice (Oryza sativa). BMC Plant Biol. 2016, 16, 134. [CrossRef] [PubMed]

17. Cao, J.; Schneeberger, K.; Ossowski, S.; Günther, T.; Bender, S.; Fitz, J.; Koenig, D.; Lanz, C.; Stegle, O.; Lippert, C.; et al. Whole-genome sequencing of multiple Arabidopsis thaliana populations. Nat. Genet. 2011, 43, 956-963. [CrossRef]

18. Cai, Q.; Li, B.; Lin, F.; Huang, P.; Guo, W.; Zheng, Y. De novo sequencing and sssembly analysis of transcriptome in Pinus bungeana Zucc. ex Endl. Forests 2018, 9, 156. [CrossRef]

19. Du, M.; Ding, G.; Cai, Q. The Transcriptomic Responses of Pinus massoniana to Drought Stress. Forests 2018, 9, 326. [CrossRef]

20. Li, Y.; Zhang, Z.; Wang, P.; Wang, S.; Ma, L.; Li, L.; Yang, R.; Ma, Y.; Wang, Q. Comprehensive transcriptome analysis discovers novel candidate genes related to leaf color in a Lagerstroemia indica yellow leaf mutant. Genes Genom. 2015, 37, 851-863. [CrossRef]

21. Lichtenthaler, H.K.; Wellburn, A.R. Determinations of total carotenoids and chlorophylls a and b of leaf extracts in different solvents. Biochem. Soc. Trans. 1983, 11, 591-592. [CrossRef]

22. Chang, S.; Puryear, J.; Cairney, J. A simple and efficient method for isolating RNA from pine trees. Plant Mol. Biol. Report. 1993, 11, 113-116. [CrossRef]

23. Salojärvi, J.; Smolander, O.-P.; Nieminen, K.; Rajaraman, S.; Safronov, O.; Safdari, P.; Lamminmäki, A.; Immanen, J.; Lan, T.; Tanskanen, J.; et al. Genome sequencing and population genomic analyses provide insights into the adaptive landscape of silver birch. Nat. Genet. 2017, 49, 904-912. [CrossRef] [PubMed]

24. Dobin, A.; Gingeras, T.R. Comment on "TopHat2: Accurate alignment of transcriptomes in the presence of insertions, deletions and gene fusions" by Kim et al. Bioinformatics 2013, 14, 000851.

25. Livak, K.J.; Schmittgen, T.D. Analysis of Relative Gene Expression Data Using Real-Time Quantitative PCR and the 2- $\triangle \Delta \mathrm{CT}$ Method. Methods 2001, 25, 402-408. [CrossRef] [PubMed]

26. Huang, H.; Wang, S.; Jiang, J.; Liu, G.; Li, H.; Chen, S.; Xu, H. Overexpression of BpAP1 induces early flowering and produces dwarfism in Betula platyphylla x Betula pendula. Physiol. Plant. 2014, 151, 495-506. [CrossRef] [PubMed]

27. Ma, X.; Sun, X.; Li, C.; Huan, R.; Sun, C.; Wang, Y.; Xiao, F.; Wang, Q.; Chen, P.; Ma, F.; et al. Map-based cloning and characterization of the novel yellow-green leaf gene ys83 in rice (Oryza sativa). Plant Physiol. Biochem. 2017, 111, 1-9. [CrossRef] 
28. Dong, H.; Fei, G.-L.; Wu, C.-Y.; Wu, F.-Q.; Sun, Y.-Y.; Chen, M.-J.; Ren, Y.-L.; Zhou, K.-N.; Cheng, Z.-J.; Wang, J.-L.; et al. A rice virescent-yellow Leaf mutant reveals new insights into the role and sssembly of plastid caseinolytic protease in higher plants. Plant Physiol. 2013, 162, 1867-1880. [CrossRef]

29. Tanaka, R.; Koshino-Kimura, Y.; Sawa, S.; Ishiguro, S.; Okada, K.; Tanaka, A. Overexpression of chlorophyllide an oxygenase (CAO) enlarges the antenna size of photosystem II in Arabidopsis thaliana. Plant J. 2002, 26, 365-373. [CrossRef]

30. Highkin, H.R.; Boardman, N.K.; Goodchild, D.J. Photosynthetic Studies on a Pea-mutant Deficient in Chlorophyll. Plant Physiol. 1969, 44, 1310-1320. [CrossRef]

31. Polle, J.E.W.; Benemann, J.R.; Tanaka, A.; Melis, A. Photosynthetic apparatus organization and function in the wild type and a chlorophyll b -less mutant of Chlamydomonas reinhardtii. Dependence on carbon source. Planta 2000, 211, 335-344. [CrossRef] [PubMed]

32. Andersson, J.; Wentworth, M.; Walters, R.G.; Howard, C.A.; Ruban, A.; Horton, P.; Jansson, S. Absence of the Lhcb1 and Lhcb2 proteins of the light-harvesting complex of photosystem II-Effects on photosynthesis, grana stacking and fitness. Plant J. 2003, 35, 350-361. [PubMed]

33. Maxwell, K.; Johnson, G.N. Chlorophyll fluorescence-A practical guide. J. Exp. Bot. 2000, 51, 659-668. [CrossRef] [PubMed]

34. Müller, P.; Li, X.P.; Niyogi, K.K. Non-photochemical quenching. A response to excess light energy. Plant Physiol. 2001, 125, 1558-1566. [CrossRef] [PubMed]

35. Sukhov, V.; Surova, L.; Sherstneva, O.; Katicheva, L.; Vodeneev, V. Variation potential influence on photosynthetic cyclic electron flow in pea. Front. Plant Sci. 2015, 5, 766. [CrossRef] [PubMed]

36. Sukhova, E.; Mudrilov, M.; Vodeneev, V.; Sukhov, V. Influence of the variation potential on photosynthetic flows of light energy and electrons in pea. Photosynth. Res. 2017, 136, 215-228. [CrossRef]

37. Cheng, J.; Fan, P.; Liang, Z.; Wang, Y.; Niu, N.; Li, W.; Li, S. Accumulation of End Products in Source Leaves Affects Photosynthetic Rate in Peach via Alteration of Stomatal Conductance and Photosynthetic Efficiency. J. Amer. Soc. Hort. Sci. 2009, 134, 667-676. [CrossRef]

38. Liu, J.; Zhou, W.; Liu, G.; Yang, C.; Sun, Y.; Wu, W.; Cao, S.; Wang, C.; Hai, G.; Wang, Z.; et al. The Conserved Endoribonuclease YbeY Is Required for Chloroplast Ribosomal RNA Processing in Arabidopsis. Plant Physiol. 2015, 168, 205-221. [CrossRef]

39. Zhou, X.-S.; Wu, D.-X.; Shen, S.-Q.; Sun, J.-W.; Shu, Q.-Y. High photosynthetic efficiency of a rice (Oryza sativa L.) xantha mutant. Photosynthetica 2006, 44, 316-319. [CrossRef]

40. Hudson, D.; Guevara, D.R.; Hand, A.J.; Xu, Z.; Hao, L.; Chen, X.; Zhu, T.; Bi, Y.-M.; Rothstein, S.J. Rice Cytokinin GATA Transcription Factor1 Regulates Chloroplast Development and Plant Architecture. Plant Physiol. 2013, 162, 132-144. [CrossRef]

41. Sjögren, L.L.; Macdonald, T.M.; Sutinen, S.; Clarke, A.K. Inactivation of the clpC1 Gene Encoding a Chloroplast Hsp100 Molecular Chaperone Causes Growth Retardation, Leaf Chlorosis, Lower Photosynthetic Activity, and a Specific Reduction in Photosystem Content. Plant Physiol. 2004, 136, 4114-4126. [CrossRef] [PubMed]

42. Lv, X.-G.; Shi, Y.-F.; Xu, X.; Wei, Y.-L.; Wang, H.-M.; Zhang, X.-B.; Wu, J.-L. Oryza sativa Chloroplast Signal Recognition Particle 43 (OscpSRP43) Is Required for Chloroplast Development and Photosynthesis. PLoS ONE 2015, 10, e0143249. [CrossRef] [PubMed]

43. Yu, B.; Gruber, M.Y.; Khachatourians, G.G.; Zhou, R.; Epp, D.J.; Hegedus, D.D.; Parkin, I.A.P.; Welsch, R.; Hannoufa, A. Arabidopsis cpSRP54 regulates carotenoid accumulation in Arabidopsis and Brassica napus. J. Exp. Bot. 2012, 63, 5189-5202. [CrossRef] [PubMed]

44. Wan, C.M.; Li, C.M.; Ma, X.Z.; Wang, Y.; Sun, C.H.; Huang, R.; Zhong, P.; Gao, Z.Y.; Chen, D.; Xu, Z.J.; et al. GRY79 encoding a putative metallo-beta-lactamase-trihelix chimera is involved in chloroplast development at early seedling stage of rice. Plant Cell Rep. 2015, 34, 1353-1363. [CrossRef] [PubMed]

45. Miyoshi, K.; Ito, Y.; Serizawa, A.; Kurata, N. OsHAP3genes regulate chloroplast biogenesis in rice. Plant J. 2003, 36, 532-540. [CrossRef] [PubMed]

46. Perez-Ruiz, J.M. Rice NTRC Is a High-Efficiency Redox System for Chloroplast Protection against Oxidative Damage. Plant Cell 2006, 18, 2356-2368. [CrossRef] [PubMed]

47. Jiang, H.; Li, M.; Liang, N.; Yan, H.; Wei, Y.; Xu, X.; Liu, J.; Xu, Z.; Chen, F.; Wu, G.; et al. Molecular cloning and function analysis of the stay green gene in rice. Plant J. 2007, 52, 197-209. [CrossRef] 
48. Nott, A.; Jung, H.-S.; Koussevitzky, S.; Chory, J. Plastid-to-nucleus retrograde signaling. Annu. Rev. Plant Biol. 2006, 57, 739-759. [CrossRef]

49. Rossini, L.; Cribb, L.; Martin, D.J.; Langdale, J.A. The Maize Golden2 Gene Defines a Novel Class of Transcriptional Regulators in Plants. Plant Cell 2001, 13, 1231. [CrossRef]

50. Yasumura, Y.; Moylan, E.C.; Langdale, J.A. A Conserved Transcription Factor Mediates Nuclear Control of Organelle Biogenesis in Anciently Diverged Land Plants. Plant Cell 2005, 17, 1894-1907. [CrossRef]

51. Wang, P.; Korkaric, M.; Waters, M.T.; Capper, R.G.; Saunders, N.J.; Langdale, J.A. GLK transcription factors coordinate expression of the photosynthetic apparatus in Arabidopsis. Plant Cell 2009, 21, 1109-1128.

(C) 2019 by the authors. Licensee MDPI, Basel, Switzerland. This article is an open access article distributed under the terms and conditions of the Creative Commons Attribution (CC BY) license (http://creativecommons.org/licenses/by/4.0/). 